\title{
LADDER HEIGHTS, GAUSSIAN RANDOM WALKS AND THE RIEMANN ZETA FUNCTION
}

\author{
By Joseph T. Chang And Yuval Peres ${ }^{1}$ \\ Yale University and University of California, Berkeley \\ Let $\left\{S_{n}: n \geq 0\right\}$ be a random walk having normally distributed incre- \\ ments with mean $\theta$ and variance 1 , and let $\tau$ be the time at which the ran- \\ dom walk first takes a positive value, so that $S_{\tau}$ is the first ladder height. \\ Then the expected value $E_{\theta} S_{\tau}$, originally defined for positive $\theta$, may be \\ extended to be an analytic function of the complex variable $\theta$ throughout \\ the entire complex plane, with the exception of certain branch point sin- \\ gularities. In particular, the coefficients in a Taylor expansion about $\theta=0$ \\ may be written explicitly as simple expressions involving the Riemann \\ zeta function. Previously only the first coefficient of the series developed \\ here was known; this term has been used extensively in developing ap- \\ proximations for boundary crossing problems for Gaussian random walks. \\ Knowledge of the complete series makes more refined results possible; we \\ apply it to derive asymptotics for boundary crossing probabilities and the \\ limiting expected overshoot.
}

1. Introduction. Let $X_{1}, X_{2}, \ldots$ be independent and distributed as $N(\theta, 1)$, the normal distribution with mean $\theta$ and variance 1 . Consider the random walk $\left\{S_{n}: n \geq 0\right\}$ consisting of the partial sums $S_{n}=X_{1}+\cdots+X_{n}$. The first time $\tau=\inf \left\{n: S_{n}>0\right\}$ that the random walk is positive is called the first ladder epoch, and the first positive value $S_{\tau}$ taken by the random walk is called the first ladder height.

The Riemann zeta function $\zeta$ is originally defined for $\operatorname{Re}(z)>1$ by the series $\zeta(z)=\sum_{n=1}^{\infty} n^{-z}$. This definition is extended by analytic continuation to the entire complex plane except $z=1$, where $\zeta$ has a simple pole. For example, the extension to $\operatorname{Re}(z)<0$ may be seen from Riemann's functional equation [see (19)], which expresses $\zeta(z)$ in terms of $\zeta(1-z)$. Calculation of the zeta function is routine; it is implemented in the computer package Mathematica, for example.

For positive $\theta$, the expected value of $S_{\tau}$ depends on $\theta$; let us write it as $E_{\theta} S_{\tau}$ to indicate this dependence. The main subject of this paper is the behavior of $E_{\theta} S_{\tau}$ near $\theta=0$. In particular, we show that the function $\theta \mapsto E_{\theta} S_{\tau}$ can be continued analytically to a neighborhood of the origin, and we find its Taylor expansion about $\theta=0$. The next theorem is our main result.

Received September 1995; revised J uly 1996.

${ }^{1}$ Research partially supported by NSF Grant DMS-94-04391.

AMS 1991 subject classifications. 60J 15, 30B40.

Key words and phrases. Random walk, ladder height, Riemann zeta function, boundary crossing probability, analytic continuation. 
THEOREM 1.1. The expected first ladder height $E_{\theta} S_{\tau}$ may be extended to be an analytic function in a neighborhood of $\theta=0$; it has the expansion

$$
E_{\theta} S_{\tau}=\frac{1}{\sqrt{2}} \exp \left\{\frac{-\theta}{\sqrt{2 \pi}} \sum_{n=0}^{\infty} \frac{\zeta\left(\frac{1}{2}-n\right)}{n !(2 n+1)}\left(\frac{-\theta^{2}}{2}\right)^{n}\right\}
$$

in the disk $\{|\theta|<2 \sqrt{\pi}\}$.

Previously only the $n=0$ term in the expansion of Theorem 1.1 was known explicitly. We will also determine the extent to which the function $\theta \mapsto E_{\theta} S_{\tau}$ can be analytically continued throughout the complex plane. It turns out that the function has branch points at $\{\sqrt{2 \pi k} \pm i \sqrt{2 \pi k}: k \neq 0, k$ integer $\}$; in particular, it can be analytically continued to a neighborhood containing the real axis.

Our starting point is a classical expression for $E_{\theta} S_{\tau}$ from the fluctuation theory of random walks; see (5). As $\theta \downarrow 0$, the series in (5) converges ever more slowly, whereas the convergence in the Taylor expansion (1) that we shall derive from it becomes more rapid. The behavior of $E_{\theta} S_{\tau}$ as $\theta \downarrow 0$ is of particular significance for applications such as heavy traffic limit theorems for queues [see, e.g., Whitt (1974) and Asmussen (1987)] and the corrected diffusion approximations of Siegmund (1979).

Theorem 1.1 is based on a result (see Theorem 2.1 in Section 2) about power series of the form $\sum_{n=1}^{\infty} n^{-\alpha} e^{-n w}$, which also arise in other contexts. For each $\alpha$, this series represents an analytic function of $w$ in the right half-plane $\{\operatorname{Re}(w)>0\}$. We investigate the analytic continuation of this function; we are particularly interested in continuing to a neighborhood of $w=0$. Most of the work was in fact done by Hardy (1905), who investigated certain questions about power series, including the behavior of the series $\sum_{n=1}^{\infty} n^{-\alpha} z^{n}$ about the point $z=1$. So our problem involves a reparametrization of Hardy's. This reparametrization turns out to be felicitous, in that the resulting expansion has a neat and explicit expression in terms of the zeta function: whereas Hardy stopped with the first coefficient in his expansion, we obtain explicit expressions for all of the coefficients in ours. To keep the present paper selfcontained, some of our development will closely follow Hardy's.

For simplicity, our treatment here is confined to random walks with Gaussian increments, which is the case that has been the most important in applications. The Gaussian case also plays a central role in the theory; a heuristic sense of this may be obtained by combining the central limit theorem with the basic identity (5) below, which holds for general increment distributions. An intricate analysis in this spirit was carried through by Lai (1976), who analyzed more general increment distributions-although with the additional restriction of zero mean-by expanding around the Gaussian case. Likewise, the importance of the series $\sum_{n=1}^{\infty} n^{-\alpha} z^{n}$ goes beyond the Gaussian case; indeed a two-term asymptotic expansion of this series as $z$ increases to 1 forms a cornerstone of the analysis of Lai (1976). 
Moments of the first ladder height have been studied in the general setting of an exponential family of distributions $\left\{F_{\theta}: \theta \in \Theta\right\}$, which may be written in the form $F_{\theta}(d x)=e^{\theta x-\psi(\theta)} F_{0}(d x)$. For example, the normal family $\{N(\theta, 1): \theta \in \mathbb{R}\}$ under consideration here is a special case having $\psi(\theta)=\theta^{2} / 2$. Let $P_{\theta}$ and $E_{\theta}$ denote probability and expectation when the distribution of $X_{i}$ is $F_{\theta}$. Under the assumptions that $\Theta$ contains an interval around $0, E_{0} X_{1}=0$, and the distribution $F_{0}$ is nonlattice, Siegmund (1979) showed that for $a>0$,

$$
E_{\theta} S_{\tau}^{a}=E_{0} S_{\tau}^{a}+\frac{a}{a+1}\left(E_{0} S_{\tau}^{a+1}\right) \theta+o(\theta)
$$

as $\theta \downarrow 0$. The question of whether the remainder $o(\theta)$ in (2) is actually $O\left(\theta^{2}\right)$ was left open by Siegmund's development. Under the slightly strengthened hypothesis that the distribution $F_{0}$ is strongly nonlattice, Chang (1992) replaced the $o(\theta)$ by $c_{a} \theta^{2}+O\left(\theta^{3}\right)$. Here $c_{a}$ is a constant defined in terms of $F_{0}$ whose general form is rather complicated, but it takes a simple form when $a=1$ and the distribution $F_{0}$ is symmetric about 0 . For the normal family, these previous results gave

$$
E_{\theta} S_{\tau}=\frac{1}{\sqrt{2}} \exp \left[\rho \theta+O\left(\theta^{3}\right)\right],
$$

where $\rho=E_{0}\left(S_{\tau}^{2}\right) /\left(2 E_{0} S_{\tau}\right) \doteq 0.583$.

The quantity $\rho$ has an interesting history. It arose in a paper of Chernoff (1965), who studied discrete and continuous versions of the problem of sequentially testing whether the drift of a Brownian motion is positive. In the discrete version corresponding to a given $\delta>0$, the Brownian motion is observed only at times $0, \delta, 2 \delta, \ldots$. Both the discrete and continuous versions have optimal solutions of the form: stop the Brownian motion at the first observation that crosses a certain boundary. Denoting the optimal boundaries for the discrete problem and the continuous problem by $x_{\delta}=x_{\delta}(t)$ and $x=x(t)$, respectively, Chernoff (1965) showed, in his notation, that

$$
x_{\delta}(t)=x(t)+\hat{z} \sqrt{\delta}+o(\sqrt{\delta}) \quad \text { as } \delta \downarrow 0 .
$$

It turns out that the number $\hat{z}$ is in fact $-\rho$. A more fundamental probabilistic interpretation of $\rho$ is as a limiting expected overshoot: defining the overshoot $R_{b}=S_{\tau(b)}-b$, where $\tau(b)$ is the first passage time inf $\left\{n: S_{n}>b\right\}$, standard results from renewal theory say that $R_{b}$ converges in distribution to a random variable $R_{\infty}$, and $E_{0} R_{\infty}=E_{0}\left(S_{\tau}^{2}\right) /\left(2 E_{0} S_{\tau}\right)$. Siegmund's result (2) with $a=1$ shows that the limiting expected overshoot $\rho$ is also the coefficient of $\theta$ in the expansion of $E_{\theta} S_{\tau} / E_{0} S_{\tau}$ as $\theta \downarrow 0$. As for numerical computation, Chernoff (1965) and Siegmund (1979) expressed $\rho$ as an integral and Lai (1976) expressed $\rho$ as the sum of a series. At the time their work was done, Lai and Siegmund were not aware of the connection to the work of Chernoff; Hogan (1986) explained the connection between the solution of Chernoff's problem and the limiting expected overshoot.

As far as we are aware, the only previous observation that relates Gaussian random walks and the zeta function is the tantalizing final sentence of the 
following quotation from Chernoff (1965) about his result (4):

This result involves relating the original problem to an associated problem and studying the limiting behavior of the solution of the associated problem. This solution corresponds to the solution of a Wiener-Hopf equation. Results of Spitzer can be used to characterize the solution of the WienerHopf equation and yield $\hat{z}$ as an integral, which, as Gordon Latta pointed out to the author, is equal to $\zeta(1 / 2) /(2 \pi)^{1 / 2}=$ -0.5824 .

[References omitted. Also, the quoted numerical value is slightly incorrect; to 4 decimal places it should be -0.5826 .] That $\rho=-\zeta(1 / 2) /(2 \pi)^{1 / 2}$ can be seen by comparing (3) and the expansion in Theorem 1.1. Lai (1976) partially rediscovered the expansion of Hardy by a different method. However, Lai's development recovered only the same terms that Hardy gave explicitly, and did not establish analyticity or relate the results to the zeta function.

The first ladder height plays a fundamental role in the theory and applications of random walks and renewal theory; see, for example, the treatises of Asmussen (1987), Feller (1971), Prabhu (1980) and Siegmund (1985), all of which feature ladder variables prominently. The particular area of application that originally motivated this investigation is the asymptotic approximation of boundary crossing probabilities for random walks. In addition to certain areas of applied probability such as queueing theory [e.g., Asmussen (1987)] and insurance risk analysis [Grandell (1991)], such boundary crossing problems arise in statistics from the study of sequential procedures, which typically sample until a certain random walk crosses a certain boundary; see Siegmund (1985) and Woodroofe (1982). They also arise in some nonsequential procedures such as likelihood ratio tests for a change point [e.g., Siegmund (1986)]. Here the likelihood ratio involves a random walk indexed by the possible change points and the test rejects if the maximum of the likelihood ratio exceeds some level, so that significance levels and $P$-values are boundary crossing probabilities. Some of the most useful and accurate approximations are derived by taking a limit as the drift of the random walk tends to zero and the boundary tends to infinity. Knowledge of the expected amount by which a random walk overshoots a high level is a basic requirement for the development of this theory. Since this limiting expected overshoot is $E_{\theta}\left(S_{\tau}^{2}\right) /\left(2 E_{\theta} S_{\tau}\right)$, to obtain asymptotics for the overshoot as $\theta \rightarrow 0$, the behavior of moments of the first ladder height as $\theta \rightarrow 0$ must be understood.

The analytic continuation and Taylor expansion of $\log E_{\theta} S_{\tau}$ are developed in the next section. In the final section we discuss some simple applications to the study of boundary crossing probabilities.

2. Analytic continuation of $E_{\theta} S_{\tau}$. The relation

$$
\log E_{\theta} \tau=\sum_{n=1}^{\infty} \frac{1}{n} P_{\theta}\left\{S_{n} \leq 0\right\} \text { for } \theta>0
$$


is a standard fact in the fluctuation theory of random walks; see, for example, Theorem 3 on page 416 of Feller (1971). Wald's equation $E_{\theta} S_{\tau}=\theta E_{\theta} \tau$ gives

$$
E_{\theta} S_{\tau}=\theta \exp \left(\sum_{n=1}^{\infty} \frac{1}{n} P_{\theta}\left\{S_{n} \leq 0\right\}\right)
$$

Differentiating (5) with respect to $\theta$, which can be justified by dominated convergence, and using normality of $S_{n}$, we obtain

$$
\frac{d}{d \theta} \log E_{\theta} S_{\tau}=\frac{1}{\theta}-\frac{1}{\sqrt{2 \pi}} \sum_{n=1}^{\infty} \frac{\left(\exp \left(-\theta^{2} / 2\right)\right)^{n}}{\sqrt{n}}
$$

for $\theta>0$. This motivates our study of series of the form $\sum n^{-\alpha} e^{-n w}$; we are particularly interested in the case $\alpha=1 / 2$ and $w=\theta^{2} / 2$, and we would like to determine the behavior of the series around $w=0$. This is done in Theorem 2.1.

Notation. It will be convenient to define $c(\alpha)=2 \pi i / \Gamma(1-\alpha)$. Since the Gamma function $\Gamma(z)$ is analytic at all $z \in \mathbb{C}$ except the points $z=$ $0,-1,-2, \ldots$, where it has simple poles, it follows that $c(\alpha)$ is an entire function, with zeros at positive integer values of $\alpha$. From the relation $\Gamma(\alpha) \Gamma(1-\alpha)=$ $\pi / \sin (\pi \alpha)$ we obtain the alternative expression $c(\alpha)=2 i \Gamma(\alpha) \sin (\pi \alpha)$ for all complex $\alpha$ other than the nonpositive integers $0,-1,-2, \ldots$.

ThEOREM 2.1. For each $\alpha \in \mathbb{C}$, the function

$$
H_{\alpha}(w):=c(\alpha) \sum_{n=1}^{\infty} n^{-\alpha} e^{-n w}-2 \pi i w^{\alpha-1},
$$

which is analytic in the half-plane $\{\operatorname{Re}(w)>0\}$, may be analytically continued to the disk $\{|w|<2 \pi\}$. In that disk, for each $\alpha \in \mathbb{C}$ other than the positive integers, $H_{\alpha}$ has the Taylor series expansion

$$
H_{\alpha}(w)=c(\alpha) \sum_{n=0}^{\infty} \zeta(\alpha-n) \frac{(-w)^{n}}{n !} .
$$

REMARK. With the appropriate interpretation, (8) holds also when $\alpha$ is a positive integer. In this case, the product $c(\alpha) \zeta(\alpha-n)$ should be interpreted as 0 , except for the case $n=\alpha-1$, where one uses the fact that $c(\alpha) \zeta(\alpha-n)$ has a removable singularity at $\alpha=n+1$.

Theorem 1.1 is an easy consequence: taking $\alpha=1 / 2$ and $w=\theta^{2} / 2$ and using the fact that $\Gamma(1 / 2)=\sqrt{\pi}$ (so that $c(1 / 2)=2 i \sqrt{\pi}$ ) gives

$$
\sum_{n=1}^{\infty} \frac{\left(\exp \left(-\theta^{2} / 2\right)\right)^{n}}{\sqrt{n}}-\sqrt{2 \pi}\left(\theta^{2}\right)^{-1 / 2}=\sum_{n=0}^{\infty} \frac{\zeta(1 / 2-n)}{n !}\left(\frac{-\theta^{2}}{2}\right)^{n} .
$$

Combining this with (6) we get

$$
\frac{d}{d \theta} \log E_{\theta} S_{\tau}=\frac{-1}{\sqrt{2 \pi}} \sum_{n=0}^{\infty} \frac{\zeta(1 / 2-n)}{n !}\left(\frac{-\theta^{2}}{2}\right)^{n} .
$$


Theorem 1.1 is obtained by integrating, exponentiating and using the fact that $E_{0} S_{\tau}=1 / \sqrt{2}$.

Proof of Theorem 2.1. Removing the ray $\mathbb{R}_{-}=\{x \in \mathbb{R}: x \leq 0\}$ from the complex plane leaves a domain $\mathbb{C} \backslash \mathbb{R}_{-}$on which the logarithm function may be defined to be single-valued and analytic by

$$
\log u=\log |u|+i \arg (u),
$$

where $-\pi<\arg (u)<\pi$, say. We then adopt the definition $u^{z}=e^{z \log u}$; for each $z \in \mathbb{C}$ this is also an analytic function of $u$ for $u \in \mathbb{C} \backslash \mathbb{R}_{-}$. Having fixed a branch of the logarithm, certain operations require a bit of care; for example, a fact that we will use below is

$$
(x y)^{z}=x^{z} y^{z} \quad \text { if }|\arg (x)+\arg (y)|<\pi .
$$

For small positive $\delta$, let $C_{\delta}$ denote the contour

$C_{\delta}=\left\{x+i \delta:-\delta^{-1} \leq x \leq 0\right\}+\left\{\delta e^{i \theta}: \pi / 2 \geq \theta \geq-\pi / 2\right\}+\left\{x-i \delta: 0 \geq x \geq-\delta^{-1}\right\}$,

traversed as shown in the dashed curve in Figure 1. Here the addition of contours indicates successive traversal in the positive direction (i.e., whatever direction the contour had as originally defined). Taking $a>0$ and $b \in(0,2 \pi)$, let

$$
\begin{aligned}
L_{\delta}= & \left\{-\delta^{-1}+i y:-\delta \geq y \geq b-2 \pi\right\}+\left\{x+i(b-2 \pi):-\delta^{-1} \leq x \leq a\right\} \\
& +\{a+y i: b-2 \pi \leq y \leq b\}+\left\{x+b i: a \geq x \geq-\delta^{-1}\right\} \\
& +\left\{-\delta^{-1}+i y: b \geq y \geq \delta\right\}
\end{aligned}
$$

this is the solid contour in Figure 1. Further, let

$$
\begin{aligned}
L= & \{x+i(b-2 \pi):-\infty<x \leq a\}+\{a+y i: b-2 \pi \leq y \leq b\} \\
& +\{x+b i: a \geq x>-\infty\}
\end{aligned}
$$

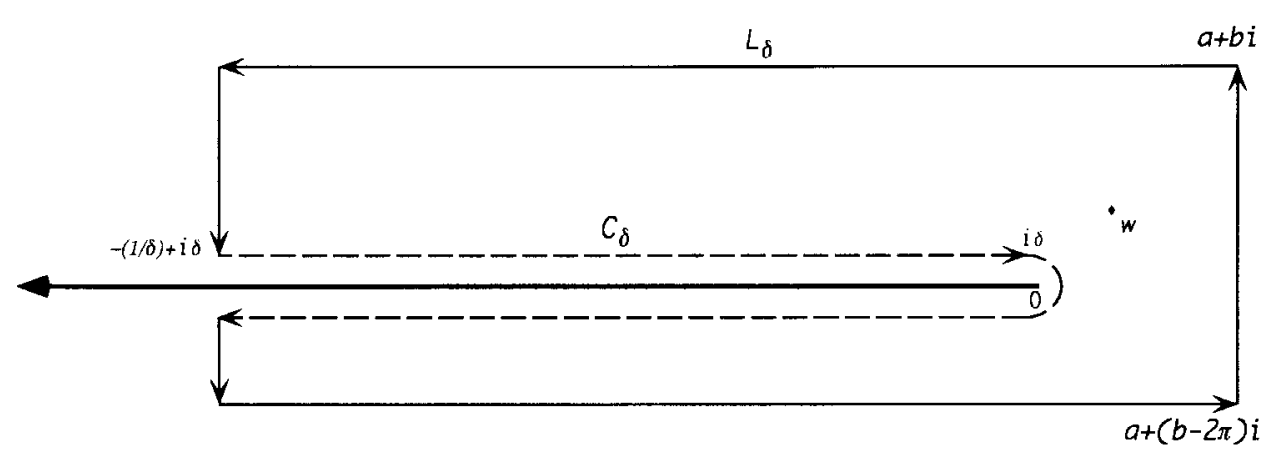

FIG. 1. 


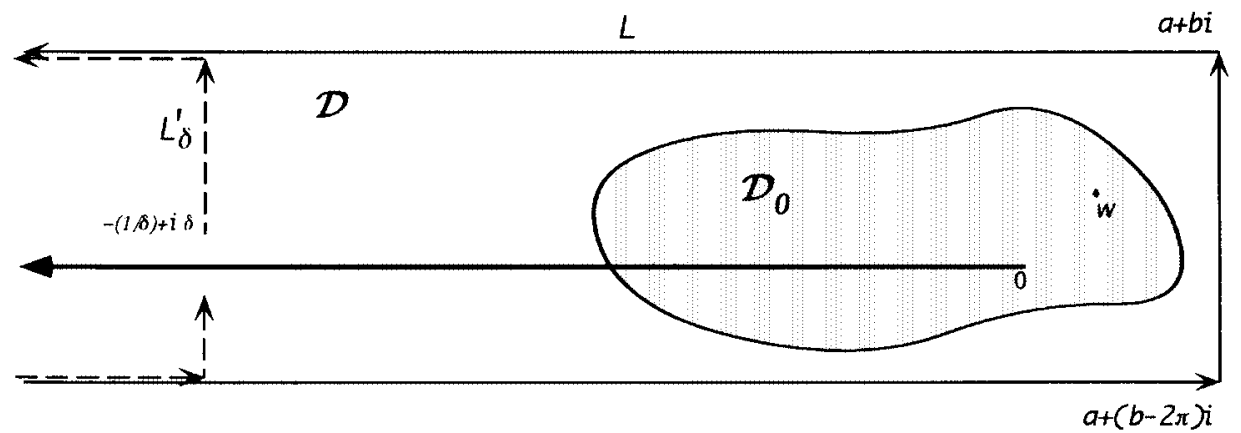

FIG. 2.

and let $L_{\delta}^{\prime}$ denote the difference $L-L_{\delta}$, as shown in Figure 2. Finally, let $\mathscr{D}$ denote the domain

$$
\mathscr{D}=\{u: \operatorname{Re}(u)<a \text { and } b-2 \pi<\operatorname{Im}(u)<b\},
$$

bounded by $L$, and let $\mathscr{D}_{0}$ be a bounded domain containing the point 0 such that the closure of $\mathscr{D}_{0}$ is contained in $\mathscr{D}$.

Lemma 2.2 (Hardy). Let $H_{\alpha}$ be as defined in (7), and define

$$
I_{\alpha}(w):=e^{-w} \int_{L} \frac{u^{\alpha-1}}{e^{-u}-e^{-w}} d u .
$$

Then for each $a \in \mathbb{C}, H_{\alpha}(w)$ is an analytic function of $w$ for $\operatorname{Re}(w)>0$, and $I_{\alpha}(w)$ is analytic for $w \in \mathscr{D}$. For all $w \in \mathscr{D} \cap\{\operatorname{Re}(w)>0\}$ and $\operatorname{Re}(\alpha)>0$ we have $H_{\alpha}(w)=I_{\alpha}(w)$.

Proof. Let $\alpha$ be arbitrary. Clearly $H_{\alpha}(w)$ is an analytic function of $w$ for $\operatorname{Re}(w)>0$, since the series in the definition (7) converges uniformly in $\{\operatorname{Re}(w) \geq c\}$ for all $c>0$. Defining $\rho=\sup \left\{\operatorname{Re}(-w): w \in \mathscr{D}_{0}\right\}$, for each $\delta \in(0,1 / \rho)$ the integral

$$
\int_{L_{\delta}} \frac{u^{\alpha-1}}{e^{-u}-e^{-w}} d u
$$

is easily seen to be an analytic function of $w$ for $w \in \mathscr{D}_{0}$; for example, one could use a combination of Fubini's and Morera's theorems, or Section 2.83 of Titchmarsh (1939). Furthermore, for $w \in \mathscr{D}_{0}$,

$$
\left|\frac{u^{\alpha-1}}{e^{-u}-e^{-w}}\right| \leq \frac{\left|u^{\alpha-1}\right|}{\left|e^{-u}\right|-e^{\rho}}
$$

holds for $\operatorname{Re}(-u)>\rho$. Thus,

$$
\int_{L_{\delta}^{\prime}} \frac{u^{\alpha-1}}{e^{-u}-e^{-w}} d u \rightarrow 0 \quad \text { as } \delta \rightarrow 0,
$$


with the convergence being uniform over $w \in \mathscr{D}_{0}$. Therefore,

$$
\lim _{\delta \rightarrow 0} \int_{L_{\delta}} \frac{u^{\alpha-1}}{e^{-u}-e^{-w}} d u=\int_{L} \frac{u^{\alpha-1}}{e^{-u}-e^{-w}} d u
$$

is analytic in $w$ on the domain $\mathscr{D}_{0}$, so that, by the arbitrary nature of $\mathscr{D}_{0},(12)$ is analytic on all of $\mathscr{D}$, and hence so is $I_{\alpha}$.

Next let $\operatorname{Re}(\alpha)>0$. A change of variables in the definition $\Gamma(\alpha)=$ $\int_{0}^{\infty} x^{\alpha-1} e^{-x} d x$ gives

$$
n^{-\alpha}=\frac{1}{\Gamma(\alpha)} \int_{-\infty}^{0}|u|^{\alpha-1} e^{n u} d u
$$

for $n \geq 1$. From this, for $\operatorname{Re}(w)>0$ we obtain

$$
\sum_{n=1}^{\infty} n^{-\alpha} e^{-n w}=\frac{e^{-w}}{\Gamma(\alpha)} \int_{-\infty}^{0} \frac{|u|^{\alpha-1}}{e^{-u}-e^{-w}} d u
$$

by Fubini's theorem.

For $w$ inside the closed contour $C_{\delta}+L_{\delta}$,

$$
\int_{C_{\delta}+L_{\delta}} \frac{u^{\alpha-1}}{e^{-u}-e^{-w}} d u=-2 \pi i e^{w} w^{\alpha-1}
$$

This can be seen by integrating over a small circle about $w$, or by changing variables and using Cauchy's integral formula. Therefore, for each $w \in \mathscr{D} \backslash \mathbb{R}_{-}$, (14) holds for sufficiently small positive $\delta$.

Next observe that for $\operatorname{Re}(\alpha)>0$,

(15) $\lim _{\delta \rightarrow 0} \int_{C_{\delta}} \frac{u^{\alpha-1}}{e^{-u}-e^{-w}} d u=-2 i \sin (\pi \alpha) \int_{-\infty}^{0} \frac{|u|^{\alpha-1}}{e^{-u}-e^{-w}} d u \quad$ for $w \in \mathscr{D} \backslash \mathbb{R}_{-}$.

Indeed, since $(u \pm i \delta)^{\alpha-1} \rightarrow|u|^{\alpha-1} \exp [ \pm i \pi(\alpha-1)]$ for $u<0$ as $\delta \downarrow 0$, routine dominated convergence arguments show that for $w \in \mathscr{D} \backslash \mathbb{R}_{-}$,

$$
\lim _{\delta \downarrow} \int_{-(1 / \delta)}^{0} \frac{(u \pm i \delta)^{\alpha-1}}{e^{-(u \pm i \delta)}-e^{w}} d u=-e^{ \pm i \pi \alpha} \int_{-\infty}^{0} \frac{|u|^{\alpha-1}}{e^{-u}-e^{-w}} d u .
$$

Thus, (15) follows from the statement

$$
\int_{\pi / 2}^{-\pi / 2} \frac{\left(\delta e^{i \theta}\right)^{\alpha-1}}{e^{-\delta e^{i \theta}}-e^{-w}} i \delta e^{i \theta} d \theta \rightarrow 0 \quad \text { as } \delta \downarrow 0,
$$

which holds for $\operatorname{Re}(\alpha)>0$.

Therefore, by (12), (14) and (15),

$$
\begin{aligned}
-2 \pi i e^{w} w^{\alpha-1} & =\lim _{\delta \rightarrow 0} \int_{C_{\delta}+L_{\delta}} \frac{u^{\alpha-1}}{e^{-u}-e^{-w}} d u \\
& =-2 i \sin (\pi \alpha) \int_{-\infty}^{0} \frac{|u|^{\alpha-1}}{e^{-u}-e^{-w}} d u+\int_{L} \frac{u^{\alpha-1}}{e^{-u}-e^{-w}} d u
\end{aligned}
$$

for $w \in \mathscr{D} \backslash \mathbb{R}_{-}$and for $\operatorname{Re}(\alpha)>0$. Combining this with (13) gives $H_{\alpha}(w)=$ $I_{\alpha}(w)$ for $w \in \mathscr{D} \cap\{\operatorname{Re}(w)>0\}$ provided that $\operatorname{Re}(\alpha)>0$. 
A key idea, also used by Hardy, is to consider the analyticity of various expressions as functions of the variable $\alpha$ as well as $w$. In the proof of the theorem, we will establish certain identities first for values of $\alpha$ with large real part, then argue by analytic continuation that the identities hold for other values of $\alpha$. The next lemma prepares the way.

Lemma 2.3. Let $H_{\alpha}$ and $I_{\alpha}$ be as defined in (7) and (10). Then for each $w \in \mathscr{D} \cap\{\operatorname{Re}(w)>0\}$, the functions $\alpha \mapsto H_{\alpha}(w)$ and $\alpha \mapsto I_{\alpha}(w)$ are both entire. Also, for each nonnegative integer $k$, the $k$ th derivative

$$
I_{\alpha}^{(k)}(0)=\left.\frac{d^{k}}{d w^{k}} I_{\alpha}(w)\right|_{w=0}
$$

is an entire function of $\alpha$.

Proof. For $\operatorname{Re}(w)>0$, the sum $\sum_{1}^{\infty} n^{-\alpha} e^{-n w}$ converges uniformly over $\alpha$ in bounded sets, so the sum is an entire function of $\alpha$, and hence so is $\alpha \mapsto H_{\alpha}(w)$. For $w \in \mathscr{D}$, the integral (11) is an analytic function of $\alpha$ for small enough positive $\delta$, and the convergence in (12) is uniform over $\alpha$ in bounded sets. Therefore, $\alpha \mapsto I_{\alpha}(w)$ is entire in $\alpha$.

To prove the final assertion of the lemma we will use the expansion

$\frac{e^{-w}}{e^{-u}-e^{-w}}=\frac{1}{e^{-u}-1}+\sum_{n=1}^{\infty}\left(e^{-w}-1\right)^{n}\left\{\frac{e^{-u}}{\left(e^{-u}-1\right)^{n+1}}\right\} \quad$ for $\left|e^{-w}-1\right|<\left|e^{-u}-1\right|$.

Letting $\alpha$ be arbitrary, note that if we take $w$ in a small enough neighborhood $\mathscr{N}$ of 0 so that $\left|\left(e^{-w}-1\right) /\left(e^{-u}-1\right)\right| \leq r<1$ holds for all $w \in \mathscr{N}$ and $u \in L$, then

$$
\begin{aligned}
\int_{L}\left|u^{\alpha-1}\right| \sum_{n=0}^{\infty}\left|\frac{\left(e^{-w}-1\right)^{n}}{\left(e^{-u}-1\right)^{n+1}}\right||d u| & =\int_{L} \frac{\left|u^{\alpha-1}\right|}{\left|e^{-u}-1\right|} \sum_{n=0}^{\infty}\left|\frac{e^{-w}-1}{e^{-u}-1}\right|^{n}|d u| \\
& \leq \sum_{n=0}^{\infty} r^{n} \int_{L} \frac{\left|u^{\alpha-1}\right|}{\left|e^{-u}-1\right|}|d u|<\infty
\end{aligned}
$$

Therefore, we may interchange sum and integral to obtain

$$
\begin{aligned}
I_{\alpha}(w) & =e^{-w} \int_{L} \frac{u^{\alpha-1}}{e^{-u}-e^{-w}} d u \\
& =\int_{L} \frac{u^{\alpha-1}}{\left(e^{-u}-1\right)} d u+\sum_{n=1}^{\infty}\left(e^{-w}-1\right)^{n} \int_{L} \frac{u^{\alpha-1} e^{-u}}{\left(e^{-u}-1\right)^{n+1}} d u
\end{aligned}
$$

for $w \in \mathscr{N}$. The convergence is uniform over $w \in \mathscr{N}$.

To evaluate the derivatives $I_{\alpha}^{(k)}(0)$, write

$$
\left.\frac{d^{k}}{d w^{k}}\left(e^{-w}-1\right)^{n}\right|_{w=0}=\sum_{j=0}^{n}\left(\begin{array}{c}
n \\
j
\end{array}\right)(-j)^{k}(-1)^{n-j} ;
$$


this is 0 if $n>k$. Since (16) expresses $I_{\alpha}(w)$ as a uniformly convergent series of analytic functions in the neighborhood $\mathscr{N}$, we may differentiate term-by-term to obtain

$$
\left.\frac{d^{k}}{d w^{k}} I_{\alpha}(w)\right|_{w=0}=\sum_{n=1}^{k}\left\{\int_{L} \frac{u^{\alpha-1} e^{-u}}{\left(e^{-u}-1\right)^{n+1}} d u\right\} \sum_{j=0}^{n}\left(\begin{array}{c}
n \\
j
\end{array}\right)(-j)^{k}(-1)^{n-j} .
$$

However, for each $n>0$, the integral

$$
\int_{L} \frac{u^{\alpha-1} e^{-u}}{\left(e^{-u}-1\right)^{n+1}} d u
$$

defines an entire function of $\alpha$, again by an easy combination of Fubini's and Morera's theorems. So the desired derivatives in (17) are entire in $\alpha$.

To complete the proof of Theorem 2.1, by Lemma 2.2, for each $\alpha \in \mathbb{C}$ we have

$$
I_{\alpha}(w)=\sum_{k=0}^{\infty} I_{\alpha}^{(k)}(0) \frac{w^{k}}{k !}
$$

for all $w$ in a neighborhood of 0 . The idea will be to evaluate the derivatives $I_{\alpha}^{(k)}(0)$ by using the function $H_{\alpha}$.

Let $w \in \mathscr{D} \cap\{\operatorname{Re}(w)>0\}$. Then Lemma 2.2 gives $H_{\alpha}(w)=I_{\alpha}(w)$ for all $\alpha$ with $\operatorname{Re}(\alpha)>0$. Therefore, by Lemma 2.3 and analytic continuation, $H_{\alpha}(w)=I_{\alpha}(w)$ holds for all $\alpha \in \mathbb{C}$.

Next let $\alpha$ be arbitrary. Since we have just shown that $H_{\alpha}(w)=I_{\alpha}(w)$ for all $w \in \mathscr{D} \cap\{\operatorname{Re}(w)>0\}$, and since both $H_{\alpha}(\cdot)$ and $I_{\alpha}(\cdot)$ are analytic on $\mathscr{D} \cap\{\operatorname{Re}(w)>0\}$, for each nonnegative integer $k$ we have the equality of the $k$ th derivatives

$$
H_{\alpha}^{(k)}(w)=I_{\alpha}^{(k)}(w) \text { for } w \in \mathscr{D} \cap\{\operatorname{Re}(w)>0\} .
$$

However, by Lemma 2.2, each derivative $I_{\alpha}^{(k)}(w)$ is an analytic function of $w$ for $w \in \mathscr{D}$. Therefore, we may compute $I_{\alpha}^{(k)}(0)$ as the limit

$$
I_{\alpha}^{(k)}(0)=\lim _{w \downarrow 0} I_{\alpha}^{(k)}(w)=\lim _{w \downarrow 0} H_{\alpha}^{(k)}(w)
$$

as $w$ decreases to 0 along the real axis. Performing the last differentiation gives

$$
I_{\alpha}^{(k)}(0)=\lim _{w \downarrow 0}\left\{(-1)^{k} c(\alpha) \sum_{n=1}^{\infty} n^{-(\alpha-k)} e^{-n w}-2 \pi i(\alpha-1) \cdots(\alpha-k) w^{\alpha-k-1}\right\},
$$

which, for $\operatorname{Re}(\alpha)>k+1$, is obviously $(-1)^{k} c(\alpha) \zeta(\alpha-k)$. Finally, by Lemma 2.3 and analytic continuation, the equality $I_{\alpha}^{(k)}(0)=(-1)^{k} c(\alpha) \zeta(\alpha-k)$ holds for $\alpha$ throughout the entire complex plane, except for the (removable) singularity of $c(\alpha) \zeta(\alpha-k)$ at $\alpha=k+1$. Thus, for $\alpha$ other than positive integers, $I_{\alpha}$, which is the analytic continuation of $H_{\alpha}$ to a neighborhood of 0 , has the Taylor series claimed in the theorem. 
By the functional equation of Riemann [see, e.g., Section 4.44 of Titchmarsh (1939)], which expresses $\zeta(1-z)$ in terms of $\zeta(z)$ for $z \neq 1$,

$$
\frac{\zeta(\alpha-k)}{k !}=2^{\alpha-k} \pi^{\alpha-k-1} \cos [\pi(-\alpha+k+1) / 2] \frac{\Gamma(k+1-\alpha)}{k !} \zeta(k+1-\alpha) .
$$

Since $\Gamma(k+1-\alpha) /(k !) \sim k^{-\alpha}$ and $\zeta(k+1-\alpha) \rightarrow 1$ as $k \rightarrow \infty$, it is easy to see that the radius of convergence of the power series $\sum \zeta(\alpha-k)(-w)^{k} /(k !)$ is $2 \pi$. This proves Theorem 2.1 .

Next we investigate the complete analytic continuation of the function $\theta \mapsto$ $E_{\theta} S_{\tau}$; we will see that it may be continued to the whole plane, with countably many rays removed.

THEOREM 2.4. For each integer $J \geq 0$, the difference

$$
c(\alpha) \sum_{n=1}^{\infty} n^{-\alpha} e^{-n w}-2 \pi i \sum_{j=-J}^{J}(w-2 \pi j i)^{\alpha-1},
$$

which is analytic in the half-plane $\{\operatorname{Re}(w)>0\}$, may be analytically continued to the disk $\{|w|<2 \pi(J+1)\}$, where it has the Taylor expansion

$$
2 i(2 \pi)^{\alpha} \sum_{k=0}^{\infty}\left(\begin{array}{c}
\alpha-1 \\
k
\end{array}\right) \sin [\pi(\alpha-k) / 2] \Delta_{J, \alpha}(k)\left(\frac{w}{2 \pi}\right)^{k},
$$

where $\Delta_{J, \alpha}(k)=\zeta(k+1-\alpha)-\sum_{j=1}^{J} j^{-(k+1-\alpha)}$.

Proof. Start by writing

$$
\Gamma(k+1-\alpha)=(-1)^{k}(\alpha-1)(\alpha-2) \cdots(\alpha-k) \Gamma(1-\alpha),
$$

so that

$$
\frac{\Gamma(k+1-\alpha)}{k !}=(-1)^{k}\left(\begin{array}{c}
\alpha-1 \\
k
\end{array}\right) \Gamma(1-\alpha)=(-1)^{k}\left(\begin{array}{c}
\alpha-1 \\
k
\end{array}\right) \frac{2 \pi i}{c(\alpha)} .
$$

Further substituting $\frac{1}{2}[\exp (i \pi(k+1-\alpha) / 2)+\exp (-i \pi(k+1-\alpha) / 2)]$ for $\cos [\pi(-\alpha+k+1) / 2]$ in (19), we obtain

$$
c(\alpha) \sum_{k=0}^{\infty} \frac{\zeta(\alpha-k)}{k !}(-w)^{k}=(2 \pi)^{\alpha}\left\{i^{\alpha} T_{-}-(-i)^{\alpha} T_{+}\right\}
$$

where

$$
T_{ \pm}=\sum_{k=0}^{\infty}\left(\begin{array}{c}
\alpha-1 \\
k
\end{array}\right)\left(\frac{ \pm i w}{2 \pi}\right)^{k} \zeta(k+1-\alpha) .
$$

For $J \geq 0$, use the definition of $\Delta_{J, \alpha}(k)$ to write

$$
T_{ \pm}=\sum_{k=0}^{\infty}\left(\begin{array}{c}
\alpha-1 \\
k
\end{array}\right)\left(\frac{ \pm i w}{2 \pi}\right)^{k} \Delta_{J, \alpha}(k)+\sum_{j=1}^{J} j^{\alpha-1} \sum_{k=0}^{\infty}\left(\begin{array}{c}
\alpha-1 \\
k
\end{array}\right)\left(\frac{ \pm i w}{2 \pi j}\right)^{k}
$$


this involves an interchange of order of summation that is easily justified if $|w|<2 \pi$. By the binomial theorem,

$$
\begin{aligned}
(2 \pi)^{\alpha} \exp (\mp i \pi \alpha / 2) \sum_{j=1}^{J} j^{\alpha-1} \sum_{k=0}^{\infty}\left(\begin{array}{c}
\alpha-1 \\
k
\end{array}\right)\left(\frac{ \pm i w}{2 \pi j}\right)^{k} & =(\mp 2 \pi i)^{\alpha} \sum_{j=1}^{J} j^{\alpha-1}\left(1 \pm \frac{i w}{2 \pi j}\right)^{\alpha-1} \\
& =\mp 2 \pi i \sum_{j=1}^{J}(w \mp 2 \pi j i)^{\alpha-1} .
\end{aligned}
$$

In the last equality we have used (9) together with the assumption that $|w|<$ $2 \pi$. Thus, for $|w|<2 \pi$,

$$
\begin{gathered}
c(\alpha) \sum_{k=0}^{\infty} \frac{\zeta(\alpha-k)}{k !}(-w)^{k}-2 \pi i \sum_{j=1}^{J}(w+2 \pi j i)^{\alpha-1}-2 \pi i \sum_{j=1}^{J}(w-2 \pi j i)^{\alpha-1} \\
=(2 \pi)^{\alpha}\left\{\exp (i \pi \alpha / 2) \sum_{k=0}^{\infty}\left(\begin{array}{c}
\alpha-1 \\
k
\end{array}\right)\left(\frac{-i w}{2 \pi}\right)^{k} \Delta_{J, \alpha}(k)\right. \\
\left.-\exp (-i \pi \alpha / 2) \sum_{k=0}^{\infty}\left(\begin{array}{c}
\alpha-1 \\
k
\end{array}\right)\left(\frac{i w}{2 \pi}\right)^{k} \Delta_{J, \alpha}(k)\right\} \\
=(2 \pi)^{\alpha} 2 i \sum_{k=0}^{\infty}\left(\frac{w}{2 \pi}\right)^{k}\left(\begin{array}{c}
\alpha-1 \\
k
\end{array}\right) \Delta_{J, \alpha}(k) \sin [\pi(\alpha-k) / 2],
\end{gathered}
$$

so that, by Theorem 2.1,

$$
\begin{aligned}
c(\alpha) & \sum_{k=0}^{\infty} n^{-\alpha} e^{-n w}-2 \pi i \sum_{j=-J}^{J}(w-2 \pi j i)^{\alpha-1} \\
& =-2 i(2 \pi)^{\alpha} \sum_{k=0}^{\infty}\left(\frac{w}{2 \pi}\right)^{k}\left(\begin{array}{c}
\alpha-1 \\
k
\end{array}\right) \Delta_{J, \alpha}(k) \sin [\pi(k-\alpha) / 2]
\end{aligned}
$$

holds for $\{|w|<2 \pi, \operatorname{Re}(w)>0\}$. For fixed $J$, simple estimates show that

$$
\Delta_{J, \alpha}(k) \sim(J+1)^{-(k+1-\operatorname{Re}(\alpha))} \quad \text { as } k \rightarrow \infty,
$$

so that the last power series has radius of convergence $2 \pi(J+1)$.

In summary, the function $G_{\alpha}(w)=c(\alpha) \sum_{n=1}^{\infty} n^{-\alpha} e^{-n w}$ can be continued throughout the complex plane except for branch point singularities at the points $2 \pi k i$ for integers $k$ (and so we must remove a ray, say $R_{k}=\{x+2 \pi k i$ : $x \leq 0\}$, from the plane for each branch point $2 \pi k i$ ). Since $G_{\alpha}$ is periodic (with period $2 \pi i)$ in the right half-plane $\{\operatorname{Re}(w)>0\}$, its analytic continuation is clearly periodic in the domain $\mathbb{C} \backslash \bigcup_{k=-\infty}^{\infty} R_{k}$. The branch point singularities of $G_{\alpha}$ are of the form $B_{\alpha, k}(w)=2 \pi i(w-2 \pi k i)^{\alpha-1}$; that is, for each $k$, the difference $G_{\alpha}-B_{\alpha, k}$ can be analytically continued to a neighborhood of $2 \pi k i$. 
The expected first ladder height involves the difference $G_{1 / 2}-B_{1 / 2,0}$ in a very simple way; in fact,

$$
\frac{d}{d \theta} \log E_{\theta} S_{\tau}=\frac{i}{2 \sqrt{2} \pi}\left\{G_{1 / 2}\left(\frac{\theta^{2}}{2}\right)-B_{1 / 2,0}\left(\frac{\theta^{2}}{2}\right)\right\} .
$$

However, we know that $G_{1 / 2}(w)-B_{1 / 2,0}(w)$ may be analytically continued to all $w$ in the domain $\mathbb{C} \backslash \bigcup_{k \neq 0} R_{k}$. Thus, $E_{\theta} S_{\tau}$ may be continued for $\theta$ throughout the complex plane, except for branch point singularities at the points $\theta=2 \sqrt{\pi k} e^{ \pm i \pi / 4}$ for nonzero integers $k$ (and the attendant rays removed). In particular, $E_{\theta} S_{\tau}$ is clearly continuable to a horizontal strip that contains the real axis.

Finally, we remark that in any fixed disk $\{|w|<c\}$, as $J$ increases, the series in (20) converges more and more rapidly, so that Theorem 2.4 provides a computationally efficient method of calculating $G_{\alpha}(w)$.

3. Applications. Perhaps the most fundamental of boundary crossing problems for random walks is determining the probability that a random walk with negative drift ever attains a positive level $b$. Letting $\tau(b)$ denote the first passage time $\inf \left\{n: S_{n}>b\right\}$, we want to approximate the probability $P_{-\theta}\{\tau(b)<\infty\}$, where $\theta>0$.

CoRollary 3.1. There exists $r>0$ such that, for $0 \leq \theta<2 \sqrt{\pi}$,

$$
P_{-\theta}\{\tau(b)<\infty\}=\exp \left\{-2 \theta\left[b-\frac{1}{\sqrt{2 \pi}} \sum_{n=0}^{\infty} \frac{\zeta\left(\frac{1}{2}-n\right)}{n !(2 n+1)}\left(\frac{-\theta^{2}}{2}\right)^{n}+O\left(e^{-r b}\right)\right]\right\}
$$

as $b \rightarrow \infty$.

Proof. The overshoot (or residual) $R_{b}$ over the level $b$ is defined by $R_{b}=$ $S_{\tau(b)}-b$ when $\tau(b)$ is finite. By Wald's likelihood ratio identity [e.g., Siegmund (1985), page 13],

$$
P_{-\theta}\{\tau(b)<\infty\}=E_{\theta}\left(\exp \left(-2 \theta S_{\tau(b)}\right)\right)=\exp (-2 \theta b) E_{\theta}\left(\exp \left(-2 \theta R_{b}\right)\right) .
$$

Under $P_{\theta}$, we have $\tau(b)<\infty$ with probability 1 , and, as $b$ tends to infinity, $R_{b}$ converges in distribution to a random variable $R_{\infty}$ having probability density $P_{\theta}\left\{S_{\tau}>x\right\} /\left(E_{\theta} S_{\tau}\right)$ with respect to Lebesgue measure for $x>0$. The expectation $E_{\theta}\left(\exp \left(-2 \theta R_{b}\right)\right)$ converges to $\nu(\theta):=E_{\theta}\left(\exp \left(-2 \theta R_{\infty}\right)\right)$, where we have adopted the notation $\nu$ used by Siegmund. Corollary 2.3 of Chang (1992) shows that this convergence is exponentially fast: for $\theta^{*}>0$, there exist $C<\infty$ and $r>0$ such that

$$
\left|E_{\theta}\left(\exp \left(-2 \theta R_{b}\right)\right)-\nu(\theta)\right| \leq C \theta \exp (-r b)
$$

for all $\theta \in\left[0, \theta^{*}\right]$ and all $b \geq 0$. The fact that $\theta^{*}>0$ may be chosen arbitrarily follows from the fact that the family of distributions $\left\{N(\theta, 1): 0 \leq \theta \leq \theta^{*}\right\}$ is uniformly strongly nonlattice, that is,

$$
\inf _{0 \leq \theta \leq \theta^{*}} \inf _{|t| \geq \delta} \mid 1-E_{\theta}(\exp (\text { it } X)) \mid>0 \text { for all } \delta>0 .
$$


We have

$$
\nu(\theta)=\frac{1}{E_{\theta} S_{\tau}} \int_{0}^{\infty} \exp (-2 \theta x) P_{\theta}\left\{S_{\tau}>x\right\} d x=\frac{1}{E_{\theta} S_{\tau}} \frac{1}{2 \theta}\left[1-E_{\theta}\left(\exp \left(-2 \theta S_{\tau}\right)\right)\right] .
$$

However, letting $\tau_{-}$denote the first descending ladder epoch $\inf \left\{n: S_{n} \leq 0\right\}$, Wald's likelihood ratio identity, symmetry of the normal distribution and duality [e.g., Corollary 8.39 of Siegmund (1985)] give

$$
1-E_{\theta}\left(\exp \left(-2 \theta S_{\tau}\right)\right)=1-P_{-\theta}\{\tau<\infty\}=P_{\theta}\left\{\tau_{-}=\infty\right\}=1 /\left(E_{\theta} \tau\right),
$$

so that

$$
\nu(\theta)=\frac{1}{\left(E_{\theta} S_{\tau}\right)\left(2 \theta E_{\theta} \tau\right)}=\frac{1}{2\left(E_{\theta} S_{\tau}\right)^{2}} .
$$

From this, our Taylor series for $\log E_{\theta} S_{\tau}$ may be transformed into a Taylor series for $\log \nu(\theta)$ about $\theta=0$, resulting in

$$
\nu(\theta)=\exp \left\{\theta \sqrt{\frac{2}{\pi}} \sum_{n=0}^{\infty} \frac{\zeta\left(\frac{1}{2}-n\right)}{n !(2 n+1)}\left(\frac{-\theta^{2}}{2}\right)^{n}\right\} .
$$

Combining this with (22) and (23) proves the corollary.

For example, in a diffusion normalization, which assumes that $\theta \rightarrow 0$ and $b \rightarrow \infty$ with $\theta b \asymp 1$, clearly the error term $O\left(e^{-r b}\right)$ is dominated by each power of $\theta$. More generally, we could let $\theta \rightarrow 0$ and $b \rightarrow \infty$ much slower than in a diffusion normalization, as in the following statement.

Corollary 3.2. If $\theta \downarrow 0$ and $b \rightarrow \infty$ in such a way that

$$
\frac{b}{\log (1 / \theta)} \rightarrow \infty
$$

then

$$
P_{-\theta}\{\tau(b)<\infty\} \approx \exp \left\{-2 \theta\left[b-\frac{1}{\sqrt{2 \pi}} \sum_{n=0}^{\infty} \frac{\zeta\left(\frac{1}{2}-n\right)}{n !(2 n+1)}\left(\frac{-\theta^{2}}{2}\right)^{n}\right]\right\}
$$

The meaning of the last display is as an asymptotic expansion in the sense of Poincaré; that is, for each $N$,

$$
P_{-\theta}\{\tau(b)<\infty\}=\exp \left\{-2 \theta\left[b-\frac{1}{\sqrt{2 \pi}} \sum_{n=0}^{N} \frac{\zeta\left(\frac{1}{2}-n\right)}{n !(2 n+1)}\left(\frac{-\theta^{2}}{2}\right)^{n}+O\left(\theta^{2 N+2}\right)\right]\right\} .
$$

Siegmund (1985) discusses further applications of the function $\nu$. The expansion (24) completes the result $\nu(\theta)=e^{-\rho \theta}+o\left(\theta^{2}\right)$ given in (4.38) of Siegmund (1985).

Other results that follow easily from Theorem 2.1 are explicit expressions for the moments $E_{0}\left(S_{\tau}^{p}\right)$. These may be found by calculating along the lines shown by Lai (1976), using our expansion of the series $\sum n^{-\alpha} e^{-n w}$ about $w=0$; 
here the series with values of $\alpha$ other than $\alpha=1 / 2$ arise. For example, the results for the third and fourth moments are

$$
E_{0}\left(S_{\tau}^{3}\right)=\frac{3}{\sqrt{2}}\left(\frac{1}{4}+\frac{\zeta^{2}(1 / 2)}{2 \pi}\right)=1.25035
$$

and

$$
E_{0}\left(S_{\tau}^{4}\right)=\frac{\zeta(3 / 2)}{\pi^{3 / 2}}-\frac{\zeta^{3}(1 / 2)}{\pi^{3 / 2}}-\frac{3 \zeta(1 / 2)}{2 \sqrt{\pi}}=2.26433 .
$$

For another example of information that can be extracted from Theorem 1.1, define the function $\rho(\theta)=E_{\theta} R_{\infty}$; note that the value of $\rho(0)$ is the number $-\zeta(1 / 2) / \sqrt{2 \pi}$ we have been calling $\rho$. The first derivative $\rho^{\prime}(0)=1 / 4$ is known. The second derivative $\rho^{\prime \prime}(0)$ can be obtained by equating the expansion (24) for the function $\nu(\theta)$ with its definition $E_{\theta}\left(\exp \left(-2 \theta R_{\infty}\right)\right)$, writing the Taylor expansion of the exponential up to order $\theta^{2}$, and using (2). The result of this calculation, which also makes use of the moments $E_{0}\left(S_{\tau}^{3}\right)$ and $E_{0}\left(S_{\tau}^{4}\right)$ given in the previous paragraph, is

$$
\rho^{\prime \prime}(0)=\frac{\zeta(3 / 2)}{2(2 \pi)^{3 / 2}}=0.08293
$$

CONCLUDING REMARKS.

1. An Associate Editor raised a question that we had also puzzled over ourselves: for negative $\theta$, is there a nice probabilistic interpretation of the analytic continuation of $E_{\theta} S_{\tau}$ ? In particular, one might entertain $E_{\theta}\left(S_{\tau} ; \tau<\right.$ $\infty)$ and $E_{\theta}\left(S_{\tau} \mid \tau<\infty\right)$ as plausible candidates. In fact neither is correct. By inspection of (1), the analytic continuation may be expressed as $1 /\left(2 E_{-\theta} S_{\tau}\right)$ for $\theta \leq 0$. Differentiating the Wiener-Hopf factorization

$\left[1-E_{\theta}\left(\exp \left(i \lambda S_{\tau}\right) ; \tau<\infty\right)\right]\left[1-E_{\theta}\left(\exp \left(i \lambda S_{\tau_{-}}\right) ; \tau_{-}<\infty\right)\right]=1-E_{\theta}\left(\exp \left(i \lambda X_{1}\right)\right)$ twice with respect to $\lambda$ and setting $\lambda=0$ shows that

$$
\frac{1}{2 E_{-\theta} S_{\tau}}=\frac{E_{\theta}\left(S_{\tau} ; \tau<\infty\right)}{1+2 \theta \rho(-\theta)+\theta^{2}}
$$

for $\theta \leq 0$, where the function $\rho$ is as defined in the previous paragraph. This is easily seen to lie strictly between $E_{\theta}\left(S_{\tau} ; \tau<\infty\right)$ and $E_{\theta}\left(S_{\tau} \mid \tau<\infty\right)$ as $\theta \uparrow 0$.

2. After this work was done we learned of another occurrence of $\zeta(1 / 2)$ in a related problem; see Asmussen, Glynn, and Pitman (1995).

Acknowledgments. Weare grateful to David Siegmund for passing along the "interesting numerical curiosity" $\rho=-\zeta(1 / 2) / \sqrt{2 \pi}$, to Peter J ones for pointing us in the direction of Hardy, and to Pavel Etinghoff, the Associate Editor, and a referee for their helpful comments. Some of this work was done at the Institute for Elementary Studies 1995 Workshop in Pinecrest, California (supported by NSF Grant DMS-93-53149); we are grateful to Robin Pemantle for inviting us. 


\section{REFERENCES}

Asmussen, S. (1987). Applied Probability and Queues. Wiley, New York.

Asmussen, S., Glynn, P. and Pitman, J. (1995). Discretization error in simulation of onedimensional reflecting Brownian motion. Ann. Appl. Probab. 5 875-896.

Chang, J. T. (1992). On moments of the first ladder height of random walks with small drift. Ann. Appl. Probab. 2 714-738.

Chernoff, H. (1965). Sequential test for the mean of a normal distribution IV (discrete case). Ann. Math. Statist. 36 55-68.

FELLER, W. (1971). An Introduction to Probability Theory and Its Applications 2, 2nd ed. Wiley, New York.

GRANDELL, J. (1991). Aspects of Risk Theory. Springer, Berlin.

HARDY, G. H. (1905). A method for determining the behavior of certain classes of power series near a singular point on the circle of convergence. Proc. London Math. Soc. 2 381-389.

Hogan, M. (1986). Comments on a problem of Chernoff and Petkau. Ann. Probab. 14 1058-1063.

LAI, T. L. (1976). Asymptotic moments of random walks with applications to ladder variables and renewal theory. Ann. Probab. 4 51-66.

Prabhu, N. U. (1980). Stochastic Storage Processes: Queues, Insurance Risk, and Dams. Springer, New York.

SiEgmund, D. (1979). Corrected diffusion approximations in certain random walk problems. Adv. in Appl. Probab. 11 701-719.

Siegmund, D. (1985). Sequential Analysis: Tests and Confidence Intervals. Springer, New York.

SiEgmund, D. (1986). Boundary crossing probabilities and statistical applications. Ann. Statist. 14 361-404.

Titchmarsh, E. C. (1939). The Theory of Functions. Oxford Univ. Press.

WhitT, W. (1974). Heavy traffic limit theorems for queues: a survey. Lecture Notes in Econom. and Math. Systems 98 307-350. Springer, New York.

Woodroofe, M. (1982). Nonlinear Renewal Theory in Sequential Analysis. SIAM, Philadel phia.

DEPARTMENT OF STATISTICS

YALE UNIVERSITY

BoX 208290 YALE STATiON

New Haven, Connecticut 06520

E-MAIL: chang@stat.yale.edu
DePARTMENT OF STATistics

367 Evans HaLL

UNIVERSITY OF CALIFORNIA

BERKELEY, CALIFORNIA 94720

AND

MATHEMATICS INSTITUTE

HEBREW UNIVERSITY

GIVAT RAM, JERUSALEM 91904

ISRAEL

E-MAIL: peres@stat.berkeley.edu 